\title{
Framework for holographic scene representation and image synthesis
}

\section{Report}

Author(s):

Ziegler, Remo; Kaufmann, Peter; Peeters, Leon; Gross, Markus H.

Publication date:

2006

Permanent link:

https://doi.org/10.3929/ethz-a-006781056

Rights / license:

In Copyright - Non-Commercial Use Permitted

Originally published in:

Technical Report / ETH Zurich, Department of Computer Science 522 


\title{
A Framework for Holographic Scene Representation and Image Synthesis
}

\author{
Remo Ziegler, Peter Kaufmann, Markus Gross
}

Computer Science Department

ETH Zurich, Switzerland

e-mail: zieglerr@inf.ethz.ch

kaufmann@pop.agri.ch

grossm@inf.ethz.ch

http:/graphics.ethz.ch/ 



\title{
A Framework for Holographic Scene Representation and Image Synthesis
}

\author{
Remo Ziegler, Peter Kaufmann, Markus Gross \\ ETH Zurich, Switzerland
}

\begin{abstract}
We present a framework for the holographic representation and display of graphics objects. As opposed to traditional graphics representations, our approach reconstructs the light wave reflected or emitted by the original object directly from the underlying digital hologram. Our holographic graphics pipeline consists of several stages including the digital recording of a full-parallax hologram, the reconstruction and propagation of its wavefront, and rendering of the final image onto conventional, framebuffer-based displays. The required view-dependent depth image is computed from the phase information inherently represented in the complex-valued wavefront. Our model also comprises a correct physical modeling of the camera taking into account optical elements, such as lens and aperture. It thus allows for a variety of effects including depth of field, diffraction, interference and features built-in anti-aliasing. A central feature of our framework is its seamless integration into conventional rendering and display technology which enables us to elegantly combine traditional 3D object or scene representations with holograms. The presented work includes the theoretical foundations and allows for high quality rendering of objects consisting of large numbers of elementary waves while keeping the hologram at a reasonable size.
\end{abstract}

Index Terms - holography, light propagation, wave, diffraction, aliasing, image synthesis, graphics representation.

\section{INTRODUCTION}

$\mathbf{T}$ RADITIONALLY, graphics objects or scenes have been represented through geometry and appearance. Most often, the former is described by geometric primitives including triangles, points, basis functions, and others, while the latter is encoded in texture maps. Due to the difficulty of representing some real-world objects, such as fur, hair, or trees, with traditional techniques, research has also focused on image based rendering using light-fields [1], [2], [3], lumigraphs [4], [5], reflectance fields [6], [7], [8], sprites, and other models. All of these graphics representations share the property that their treatment of light is motivated through ray optics and that rendering involves projection and rasterization, or ray tracing.

In recent years, significant progress has been made in the field of display technology with the goal to replace the $2 \mathrm{D}$ flat screen by more advanced and immersive 3-dimensional displays. Besides various autostereoscopic displays [9], [10], there has been an increased interest in holographic displays [11] which reconstruct the 3D object or scene wavefront directly from the underlying holographic representation. In spite of some current limitations, such as computational costs

R. Ziegler, P. Kaufmann and M. Gross are with the Computer Graphics Laboratory, ETH Zurich, Switzerland. Email: \{zieglerr, grossm\}@inf.ethz.ch kaufmann@pop.agri.ch and sampling rates, the rapid development of compute power and data storage makes holographic representation, image generation and display a technology of greatest potential for the future of computer graphics and interactive visual simulation.

In this paper we present a framework for graphics representation, processing and display which is entirely based on digital holography and wave optics. Such holograms are elegant structures capturing the phase and amplitude of an object or scene wavefront as seen from all possible views through a window of given aperture. The major difference to a lightfield, however, is its intrinsic wave optics handling anti-aliasing implicitly and its ability to reproduce object depth through phase. Thus, holograms overcome some of the inherent limitations of image based methods including defocus and compositing with conventional graphics scenes.

A central feature of this framework is its seamless integration into conventional, framebuffer-oriented 2D display technology as well as its compliance with future 3D holographic displays. The core component of our representation is a digital hologram which can be recorded both from realworld objects and from conventional, triangle or point based graphics objects. In order to reconstruct 2D images at a given camera position, the original wavefront has to be reconstructed from the hologram and propagated through space. To this end, we utilize fast, discrete approximation methods based on Fourier theory and angular spectrum. In addition, we will show that this approach allows for an elegant modeling of the camera optics. A crucial step in the rendering of digital holograms on a conventional 2D screen is the reconstruction of the depth buffer. We will present a novel algorithm to reliably reconstruct object depth from the digital hologram and to render it along with conventional graphics representations.

Despite some earlier work on holographic representations in graphics [12], [13], [14] and computer generated holography (CGH) [15], [11] this paper for the first time presents a full framework for a holography-inspired graphics pipeline, which allows to generate, process and render holograms from synthetic and real 3D objects. Rendering holograms from synthesized objects allows using small holograms, since the wavelength can be chosen outside of the visible spectrum. Our main contributions are both fundamental and practical. They include a summary of the relevant theory as well as a variety of algorithms and methods for the digital recording, wave propagation and 2D rendering of holograms. Some rendering and compositing examples demonstrate the versatility and quality of the presented methods. 


\section{RELATED WORK}

A lot of work has been done in holography since Dennis Gabor invented "wavefront reconstruction" in 1948 [16], which today is known as holography. More recently, the progress in computer technology provided the possibility to propagate light waves in space efficiently and therefore to record as well as to reconstruct a hologram digitally. Goodman [17] gives a good introduction to fast paraxial light wave propagation of planar wavefronts based on the Fourier spectrum. Restrictions concerning tilted planes are lifted in [18], [19], [20] while still applying paraxial propagation of waves. Delen and Hooker [21] extend Tommasi's system such that propagation to parallel planes becomes feasible while having the precision of Rayleigh-Sommerfeld. Fourier analysis has also successfully been applied to light transport based on ray optics by Durand et al. [22] where the influence of shading, occlusion and transport on the frequency content of radiance is studied.

Fourier spectrum propagation has recently been applied by Matsushima [23], [24] for digital hologram recording of textured objects. Similarly to the method presented in [25], the 3-D Fourier spectrum has been used for fast 2D slice projection for computer generated holography in [26]. Further efficient propagation of objects consisting of points, lines or triangles were presented in [27], [28], [29] showing a clear quality loss in the hardware based approaches. Lucente et al. [12], [13] and Halle and Kropp [14] take advantage of graphics hardware to render higher quality holographic stereograms to a holographic screen achieving interactive rates while loosening the constraint of full-parallax. An excellent overview of holographic screens is given in [11].

Digital reconstructions of captured holograms allow mathematical filtering [30], [31] improving the final image quality. Filtered holograms can adopt negative or complex values and therefore not be usable for holographic screens anymore. Still, the digital reconstruction of the filtered hologram can be achieved as shown in [32]. However, Schnars and Jüptner did not simulate a camera model with a lens showing limited depth of field. Recent interest in realistic camera models with limited depth of field has been shown in computer graphics in [3], [33], [34]. $\mathrm{Ng}$ [35] even built a prototype of a handheld plenoptic camera capturing a 4D light field of which a refocused 2D image can be calculated.

\section{OVERVIEW}

In this section a conceptual overview of the complete wave framework including our novel holographic pipeline (highlighted in orange in Fig. 1) will be given.

The first step of this framework involves recording of holograms. We distinguish between recording and acquisition of holograms. Acquisition stands for the physical capturing of a hologram of real objects onto a high resolution photographic film or a CCD as in digital holography, whereas recording (cf.Sect. V-B) will be used for the computation of a hologram from synthesized objects as in computer generated holography (CGH). In order to generate a hologram from a wavefront given on the surface of a synthetic object we have to be able to propagate this wavefront to any position and time in space. We explain different techniques of propagation in Sect. IV. Furthermore the hologram can be used as input to

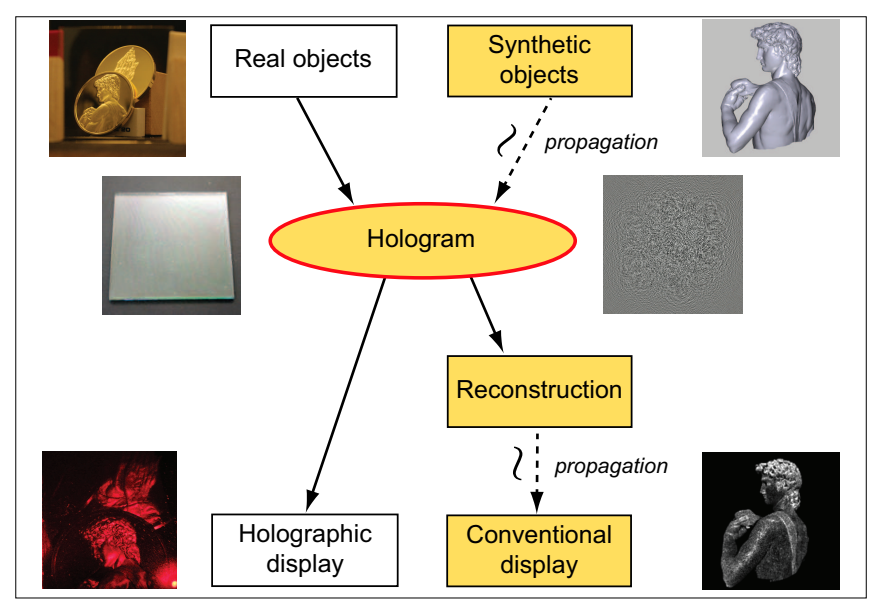

Fig. 1. Overview of the wave framework with the components of our new wave pipeline highlighted in orange. (The David dataset is courtesy of Stanford University, Digital Michelangelo Project.)

a holographic screen as well as an input for further processing (cf.Sect. VI) leading to an output on a conventional display. This output requires a discrete reconstruction of the wavefront at the hologram followed by a propagation of this wavefront to an arbitrarily placed camera as described in Sect. VII, which provides the input to a conventional display.

We will give a thorough introduction into theory of scalar waves in Sect. IV since it builds the fundamental description of how waves are traveling through space in our pipeline and can therefore be used for recording and reconstruction of holograms as well as image generation. In the remainder of the paper we concentrate on the theoretical aspects and problems of the wave pipeline and refer to [17] for issues only being relevant to practical holography.

\section{Propagation}

Light propagation is affected by the phenomena of refraction, reflection, diffraction, and interference. In our current model we do not account for refraction or reflection, but focus on the influence of diffraction and interference. We describe the evaluation of spherical waves and plane waves at arbitrary points in space in Sect. IV-A and elaborate the efficient propagation of a wavefront given on a surface $S$ to an arbitrary point $P^{\prime}$ or to an arbitrarily placed surface $S_{A^{\prime}}$ in the remainder of the section. Since most of our synthetic objects consist of planar primitives like triangles and rectangles we will focus on their propagation efficiency and refer to [27] for a fast evaluation of point sampled objects based on recurrence formula. Issues arising when evaluating the continuous equations in discrete space are discussed in Sect. IV-D.

\section{A. Scalar Wave Representation}

Throughout the paper the wave representation is based on scalar diffraction theory and is therefore an approximation of the complete Maxwell equations. A general time harmonic 
scalar wave $u(P, t)$ at position $P$ and time $t$ can be represented as

$$
u(P, t)=\Re\left\{U(P) e^{-\mathbf{i} \omega t}\right\}
$$

with $\omega=2 \pi v$ being the angular frequency while $v$ describes the frequency or oscillation of the wave. The complex function $U(P)$ with the real-valued amplitude $A(P)$ and phase $\varphi(P)$ at position $P$ is defined as

$$
U(P)=A(P) e^{\mathbf{i} \varphi(P)} .
$$

The time-dependent term $e^{-\mathbf{i} \omega t}$ of Eq.(1) can be neglected since the simulated light of our pipeline is of monochromatic nature, as is most often the case when working with holograms and therefore $U(P)$ is sufficient to adequately describe $u(P, t)$ and is used as the wave representation in our paper.

A spherical wave originating at point $P_{0}$ is being represented as

$$
U(P)=A_{0} \frac{e^{\mathbf{i} k r+\varphi_{0}}}{r}
$$

where $A_{0}$ is the real-valued amplitude at $P_{0}, k$ is the wave number, $\varphi_{0}$ the initial phase at $P_{0}, P$ is the position of evaluation and $r=\left\|P-P_{0}\right\|_{2}$. The wave number $k$ is defined as $k=\frac{2 \pi}{\lambda}$ with $\lambda$ being the wavelength. A monochromatic plane wave can be represented as

$$
U(P)=A_{0} e^{\mathrm{ik \bullet}+\varphi_{0}}
$$

where $A_{0}$ and $\varphi_{0}$ are the real-valued amplitude and phase at the origin $P_{0}$ and $\mathbf{r}$ is defined as $\mathbf{r}=P-P_{0}$. The vector $\mathbf{k}$ is defined as $\mathbf{k}=k *(\alpha, \beta, \gamma)$ with $k$ being the wave number and $(\alpha, \beta, \gamma)$ being the unit vector pointing in the direction of the wave propagation. The components of the vector are called directional cosines.

\section{B. Wavefront Propagation}

In 1678 Christian Huygens [17] presented a way of describing the propagation of a wavefront as the "envelope" of "secondary" spherical waves being emitted by every point of the primary wavefront at time $t=0$ (cf. Fig. 2). Later Gustav

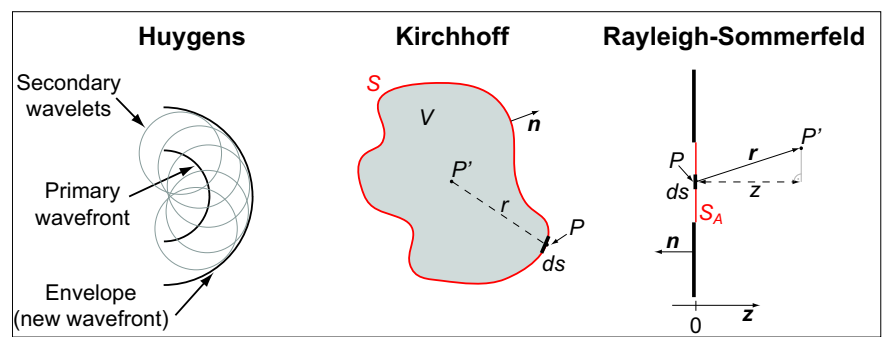

Fig. 2. Propagation of a wavefront based on Huygens, Kirchhoff's and Rayleigh-Sommerfeld's formulations.

Kirchhoff put the theory on firmer mathematical ground by finding a formulation to evaluate the wave at an arbitrary point $P^{\prime}$ in space knowing the evaluation and the derivative of the homogeneous wave equation on a closed surface $S$, enclosing volume $V$ containing $P^{\prime}$ (cf. Fig. 2):

$$
\begin{aligned}
& U\left(P^{\prime}\right)=\frac{1}{4 \pi} \iint_{S} \frac{e^{\mathbf{i} k r}}{r} \frac{\partial U(P)}{\partial \mathbf{n}}-U(P) \frac{\partial}{\partial \mathbf{n}}\left(\frac{e^{\mathbf{i} k r}}{r}\right) d s \\
& U\left(P^{\prime}\right)= \begin{cases}U\left(P^{\prime}\right) & \text { if } P^{\prime} \in V \\
0 & \text { if } P^{\prime} \notin V\end{cases}
\end{aligned}
$$

$\mathbf{n}$ is the surface normal, $U\left(P^{\prime}\right)$ is the evaluation at point $P^{\prime}$, whereas $U(P)$ is the evaluation of the wave at $P \in S$. The so-called Rayleigh-Sommerfeld theory can be derived from the Kirchhoff formula with the difference of only imposing boundary conditions on the evaluation of the wave function or on its derivative. The limitation of only being valid for planar geometries is not a real restriction, since the hologram as well as different primitives of synthetic objects like triangles and rectangles are planar as well. Assuming point $P^{\prime}$ away from the immediate vicinity $(k \gg 1 / r)$ of the aperture $S_{A}$, a propagation in the positive $\mathbf{z}$ direction perpendicular to $S_{A}$ with $S_{A}$ at $z=0$ and $P \in S_{A}$ leads to the Rayleigh-Sommerfeld formula

$$
\begin{aligned}
& U\left(P^{\prime}\right)=\frac{1}{2 \pi} \iint_{S_{A}} U(P) \frac{\partial}{\partial \mathbf{n}}\left(\frac{e^{\mathbf{i} k r}}{r}\right) d s \\
& U\left(P^{\prime}\right)=\frac{\mathbf{i}}{\lambda} \iint_{S_{A}} U(P) \frac{e^{\mathbf{i} k r}}{r}(\mathbf{r} \bullet \mathbf{n}) d s \quad, \text { with } \frac{k}{2 \pi}=\frac{1}{\lambda}
\end{aligned}
$$

The Rayleigh-Sommerfeld equation Eq.(7) can be interpreted from a physical point of view as a superposition of spherical waves $\frac{e^{\mathrm{i} k r}}{r}$ located at the aperture $S_{A}$ with amplitude $\frac{U(P)}{\lambda}$ multiplied by a phase shift of $90^{\circ}$ caused by the multiplication of $\mathbf{i}$. Additionally the spherical waves are multiplied by a directional factor $(\mathbf{r} \bullet \mathbf{n})$. For further details and derivations please see [17] and included references.

Evaluating these general forms of scalar diffraction theory can be very time consuming. There exist two approximations one for the near field called the Fresnel approximation and one for the far field called the Fraunhofer approximation. As shown in [17] the Fresnel approximation can be efficiently calculated by a convolution, while the Fraunhofer approximation can be interpreted as a Fourier transform.

However, direct integration as well as the approximations have the deficiency of either being inefficient or inaccurate. Therefore, we apply a method firstly presented by Delen and Hooker [21] and refined by Matsushima [23] where they present an accurate and fast propagation based on angular spectrum propagation presented in [36], [17].

\section{Angular Spectrum}

The complex monochromatic planar field $U(x, y, 0)$ given at an aperture $S_{A}$ can be split into multiple uniform infinite plane waves traveling in different directions (cf.Fig. 3a) by applying a Fourier transform on $U(x, y, 0)$ leading to the angular spectrum $\mathscr{A}\left(v_{x}, v_{y}, 0\right)$. Therefore, the angular spectrum $\mathscr{A}\left(v_{x}, v_{y}, 0\right)$ is an assembly of all plane waves defined as $e^{-2 \pi \mathbf{i}\left(v_{x} x+v_{y} y\right)}$ in Eq.(8). The frequencies $v_{x}$ and $v_{y}$ can be substituted by the directional $\operatorname{cosines} \alpha$ and $\beta$ of the plane 


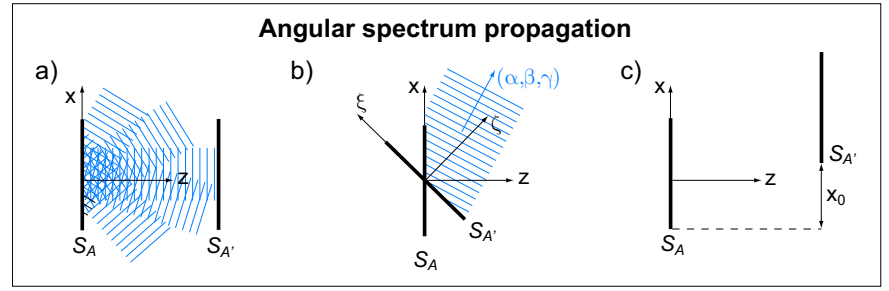

Fig. 3. The angular spectrum splits a wave field in multiple plane waves and can be used to propagate a wave from an aperture $S_{A}$ to an aperture $S_{A^{\prime}}$. For an arbitrary placement of $S_{A^{\prime}}$ "straight" propagation a), rotation of the spectrum b) and propagation on parallel offset planes c) is needed. The figure shows the $2 \mathrm{D}$ case, where the third dimension can be imagined to be perpendicular to $x$ and $z$.

wave Eq.(4) as $v_{x}=\frac{\alpha}{\lambda}$ and $v_{y}=\frac{\beta}{\lambda}$ leading to Eq.(9).

$$
\begin{aligned}
\mathscr{A}\left(v_{x}, v_{y}, 0\right) & =\int_{-\infty}^{\infty} \int_{-\infty}^{\infty} U(x, y, 0) e^{-2 \pi \mathbf{i}\left(v_{x} x+v_{y} y\right)} d x d y \\
& =\mathscr{F}\{U(x, y, 0)\} \\
\mathscr{A}\left(\frac{\alpha}{\lambda}, \frac{\beta}{\lambda}, 0\right) & =\int_{-\infty}^{\infty} \int_{-\infty}^{\infty} U(x, y, 0) e^{-2 \pi \mathbf{i}\left(\frac{\alpha}{\lambda} x+\frac{\beta}{\lambda} y\right)} d x d y
\end{aligned}
$$

To propagate the wave field along $\mathbf{z}$ to a parallel plane at distance $z$, the angular spectrum $\mathscr{A}\left(v_{x}, v_{y}, 0\right)$ has to be multiplied by a propagation phase term:

$$
\mathscr{A}\left(\frac{\alpha}{\lambda}, \frac{\beta}{\lambda}, z\right)=\mathscr{A}\left(\frac{\alpha}{\lambda}, \frac{\beta}{\lambda}, 0\right) e^{\frac{2 \pi}{\lambda} z \mathbf{i} \sqrt{1-\alpha^{2}-\beta^{2}}} \text { for } \alpha^{2}+\beta^{2}<1
$$

In case of $\alpha^{2}+\beta^{2}>1$ the wave would evaluate to a realvalued so-called evanescent wave and therefore not carry any energy away from the plane at $z=0$. The propagation defined in [17] is restricted to propagation between parallel planes sharing a common center.

1) Angular Spectrum Rotation: Using the rotation of the angular spectrum from plane $S_{A}$ with coordinate system $(x, y, z)$ to $S_{A^{\prime}}$ with coordinate system $(\xi, \eta, \zeta)$ (cf.Fig. 3 b) as proposed by [18] lifts the restriction of propagation onto parallel planes. The rotational matrix $M$ transforming $(\xi, \eta, \zeta)$ into $(x, y, z)$ also relates the corresponding spatial frequencies $\left(v_{\xi}, v_{\eta}, v_{\zeta}\right)$ to $\left(v_{x}, v_{y}, v_{z}\right)$ by

$$
\left(v_{\xi}, v_{\eta}, v_{\zeta}\right)^{T}=M^{T}\left(v_{x}, v_{y}, v_{z}\right)^{T}
$$

The coordinate transformation causes a shift of the sampling points requiring interpolation of the values for the new coordinate system. Additionally backward propagating waves are set to 0. According to [21] linear interpolation is appropriate to find amplitudes at intermediate points.

Propagation To Parallel Plane With Offset: If the parallel plane $S_{A^{\prime}}$ is offset to the propagation direction of $S_{A}$ by $\left(x_{0}, y_{0}, 0\right)$ as depicted in Fig. 3c) the propagation can be calculated by means of the Fourier shift theorem [21] as

$$
\begin{aligned}
U(x, y, z)=\int_{-\infty}^{\infty} \int_{-\infty}^{\infty} \hat{A}\left(v_{x}, v_{y}, 0\right) e^{\mathrm{i} 2 \pi z \sqrt{\frac{1}{\lambda^{2}}-v_{x}^{2}-v_{y}^{2}}} \times \\
\\
\times e^{\mathrm{i} 2 \pi\left(v_{x} x+v_{y} y\right)} d v_{x} d v_{y},
\end{aligned}
$$

where

$$
\hat{\mathscr{A}}\left(v_{x}, v_{y}, 0\right)=\mathscr{A}\left(v_{x}, v_{y}, 0\right) e^{\mathbf{i} 2 \pi\left(v_{x} x_{0}+v_{y} y_{0}\right)}
$$

expresses the Fourier shift theorem. Using the propagation of the angular spectrum in continuous space yields to the identical result as the Rayleigh-Sommerfeld (RS) formula Eq.(6). A practical implementation of RS using convolution affords a discretization leading to different problems which are treated in the following sections.

\section{Discrete Propagation}

The Fourier transform Eq.(8) and the continuous propagation Eq.(12) are defined as an integral over an infinite plane, whereas in the discrete case the Discrete Fourier Transform (DFT) and the summation is restricted to a finite size leading to artifacts. Furthermore the energy conservation does not hold, since the wave is not being evaluated over an infinite plane after propagation and therefore decreases with an increasing distance $z$ [20]. By zero-padding the plane before propagation those artifacts can be limited.

DPPO: For the sake of simplicity the derivation for discrete propagation to parallel planes with offset (DPPO) will be based on a propagation between two squares but the extension to rectangles is straightforward.

The aperture $S_{A}$ is sampled using a uniform grid of $N \times N$. The discretized wave field on $S_{A}$ is given as $U_{x y}$ and Fourier transformed using DFT leading to the angular spectrum $\mathscr{A}_{m l}$. The propagated wave field $U_{x y}^{\prime}$ can be evaluated at distance $z$ based on Eq.(12) in the discrete case as

$$
U_{x y}^{\prime}=\frac{1}{N^{2}} \sum_{m=0}^{N-1} \sum_{l=0}^{N-1} \hat{\mathscr{A}}_{m l} e^{\mathbf{i} 2 \pi z \sqrt{\frac{1}{\lambda^{2}}-\left(\frac{m}{N}\right)^{2}-\left(\frac{l}{N}\right)^{2}}} e^{\mathbf{i} 2 \pi\left(\frac{m}{N} x+\frac{l}{N} y\right)}
$$

for $x=0 . . N-1$ and $y=0 . . N-1$ with

$$
\hat{\mathscr{A}}_{m l}=\mathscr{A}_{m l} e^{\mathrm{i} 2 \pi\left(\frac{m}{N} x_{0}+\frac{l}{N} y_{0}\right)} \text {. }
$$

The asymptotic complexity can be reduced from $O\left(N^{4}\right)$ for the direct integration of RS to $O\left(N^{2} \log N\right)$ for DPPO whereas the complexity of the rotation described in Sect. IV-C.1 is negligible. The complexity of DPPO differs solely by a constant factor from the Fresnel and Fraunhofer approximation while still evaluating the propagation with the precision of RS.

Discrete Propagation Pipeline: So far we summarized one method for rotation or tilting in Sect. IV-C.1 and one for propagation in Sect. IV-D. These two methods can now be combined to evaluate a wave originating on $S_{A}$ on any arbitrarily placed aperture $S_{A^{\prime}}$. The accuracy of the result relies on the sampling of $S_{A}$ and $S_{A^{\prime}}$ and on the order of the tilt and the propagation step. In case propagation and tilt would be needed, the tilt operation has to be evaluated first. For minimal sampling requirements refer to Sect. VI-A.

Since the source aperture $S_{A}$ does not necessarily have the same resolution and size as $S_{A^{\prime}}$, re-sampling or resizing might be necessary. The four possible cases and their solution are depicted in Fig. 4. The sizes of the apertures $S_{A}$ and $S_{A^{\prime}}$ are preferably chosen as a power of 2 in order to guarantee an efficient FFT. 


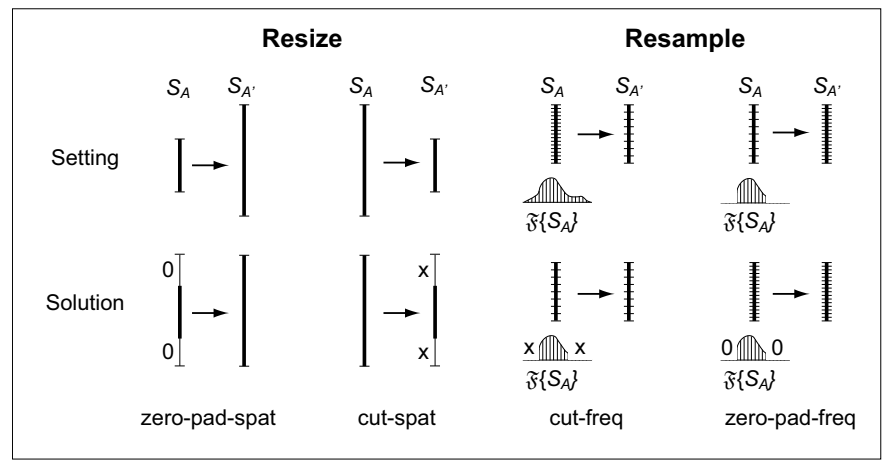

Fig. 4. The top row depicts the setting of $S_{A}$ and $S_{A^{\prime}}$ before modification. The bottom row shows the solution for the matched size after zero-padding in the spatial domain or cutting in the spatial domain and the adjusted sampling by zero-padding in the frequency domain and cutting in the frequency domain.

\section{HOLOGRAM}

\section{A. Basics}

A full-parallax hologram can be imagined as a window revealing limited viewing possibilities from a 3D scene (cf. Fig. 1). There exist many different holograms, being able to capture different properties of complex waves, such as phase, amplitude or intensity. Different setups and different shapes increase the variety even further [17]. We are simulating a transmission hologram as in Fig. 5, a setup where the laser and the object are placed on the same side of the finite planar hologram during recording. The reconstruction of this type of hologram requires a laser placed at the same relative position to the hologram and having the same wavelength as for recording. For the remainder of the paper we will refer to a transmission hologram simply as hologram. The hologram

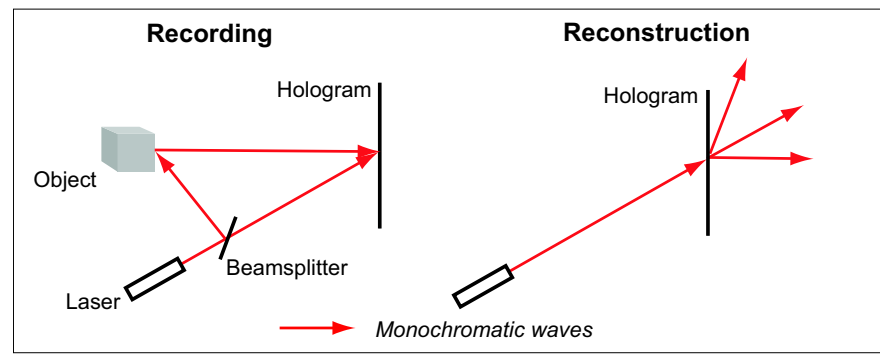

Fig. 5. Possible setup of a real transmission hologram using a beam-splitter to warrant monochromatic waves.

is a thin real-valued and densely sampled plane measuring the static wavefield intensity created by interference of multiple monochromatic waves. In a physical setup of a hologram the laser light source is used to illuminate the object as well as the hologram in order to guarantee monochromatic nature of the waves. The laser can be split up in these two parts using a beam-splitter as depicted in Fig. 5. The light from the object will be called the object wave, whereas the light from the laser during recording will be called reference wave and during reconstruction reconstruction wave. The following two sections will conceptually describe the recording of a hologram in Sect. V-B as well as the reconstruction of the object wave in Sect. VI. Capturing the interference pattern affords a very high sampling rate of the hologram. Sampling rate requirements will be discussed in Sect. VI-A.

\section{B. Recording}

For simplicity we will base the evaluation of a wave in space in this section and Sect. VI on equations described in Sect. IVA. The same concept of recording and reconstruction holds when evaluating waves as described in Sect. IV. Furthermore, having a purely theoretical approach allows assumptions of monochromatic laser properties implying spatial and temporal coherence and no motion artifacts. Two incident light waves

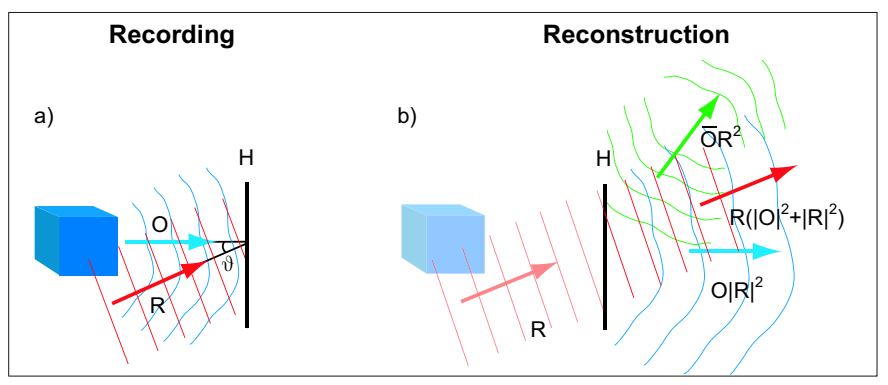

Fig. 6. (a) Hologram recording by interference of object wave $\mathrm{O}$ and reference wave R. (b) Hologram reconstruction using the reconstruction wave $\mathrm{R}$ resulting in the reference term $R\left(|O|^{2}+|R|^{2}\right)$, the real image $\bar{O} R^{2}$ and the virtual image $O|R|^{2}$.

and a holographic plate are needed to generate a hologram. One of the light waves being of type Eq.(4) stems from the laser source and will be called reference wave $R$. Another wave stems from the object and will be referred to as object wave $O$. In a theoretical recording, we do not have to simulate the reflection of the laser light at the object as depicted in Fig. 5, but sample our object into many point sources or planar segments emitting waves of type Eq.(2) with the same wavelength $\lambda$ as the laser light $R$. Therefore $O$ is the superposition of all waves emitted by the subsegments of the object. Figure 6a) shows the applied setup for hologram recording. The two light waves $O$ and $R$ are mutually coherent, leading to a time independent interference pattern in space. The intensity of this interference pattern is being recorded by a holographic plate as function $H$ :

$$
H=|O+R|^{2}
$$

Methods as presented in Sect. IV can be used to evaluate the object wave at the hologram in an efficient manner.

\section{RECONSTRUCTION}

The holographic material is assumed to provide a linear mapping between the recorded intensity and the amplitude transmitted or reflected during the reconstruction process. A reconstruction wave $R^{\prime}$ being the same as the former reference wave $R$ illuminates the holographic plate and is modulated by the transmittance $T$

$$
T=t_{b}+\beta^{\prime} H=H, \text { with } t_{b}=0 \text { and } \beta^{\prime}=1 .
$$

Thereby, $t_{b}$ is a "bias" transmittance which can be set to 0 and $\beta^{\prime}$ is a scaling factor which will be set to 1 in our theoretical 
setup leading to $T=H$. The incident reconstruction wave $R^{\prime}$ is therefore modulated by $H$ leading to a reconstructed wave $U$ as:

$$
\begin{aligned}
U=R^{\prime} \cdot H & =R^{\prime}\left(|O|^{2}+|R|^{2}\right)+O \bar{R} R^{\prime}+\bar{O} R R^{\prime} \\
& =\underbrace{R\left(|O|^{2}+|R|^{2}\right)}_{\text {reference term }}+\underbrace{O|R|^{2}}_{\text {virtual image }}+\underbrace{\bar{O} R^{2}}_{\text {real image }}
\end{aligned}
$$

Reference Term The reference term $R\left(|O|^{2}+|R|^{2}\right)$, can be approximated as a scaled version of the reference wave. This assumption is true if $|O|^{2}$ and $|R|^{2}$ are constant over the whole hologram. For $R$ being a planar wave this is trivial. However, for $O$ the assumption is only true if the object is not close to the hologram or if $|O|^{2}$ is small compared to $|R|^{2}$ and therefore variations would be negligible. The influence of $O$ is further discussed in Sect. VI-B.

Virtual Image The term $O|R|^{2}$ can be considered proportional to $O$, since $|R|^{2}$ is constant over the whole hologram. This is also called the virtual image and is the part we are interested in, since it describes the same wave as has been emitted by the object during recording.

Real Image The last term $\bar{O} R^{2}$ is the conjugate of the object wave and produces the real image which corresponds to an actual focusing of light in space. The conjugate wave $\bar{O}$ is propagating in the opposite direction of $O$ and therefore focuses in an image in front of the hologram. If $R$ is perpendicularly incident to the hologram plane, $R^{2}$ is a constant value and $\bar{O}$ will be mirrored at the hologram.

\section{A. Sampling}

Recording the interference pattern affords a very high sampling rate. It depends on the wavelength $\lambda$ and the angle $\vartheta$ between the direction of propagation of $O$ and the propagation direction of $R$. The sampling distance $d$ can be computed by

$$
d=\frac{\lambda}{2 \sin (\vartheta)}
$$

The chosen sampling distance during recording also has an influence on the maximum viewing angle of the hologram which has to be considered when positioning a camera in Sect. VII. To achieve a maximum viewing angle $\vartheta=\frac{\pi}{2}$ the sampling distance $d$ has to be at least as small as $\frac{\lambda}{2}$ in order to guarantee that the Nyquist frequency of the hologram is equal to the carrier frequency of the interference pattern. Note, that by simulating synthetic objects only three orders of magnitude bigger than the wavelength we are able to keep the hologram size reasonable and still get nice images, since the resolution of the final image and not the physical size of it is important when rendering to a screen (cf. Tab. I).

\section{B. Filtering}

The reconstruction leads to three different terms (cf. Fig. 6b), whereas we are only interested in the virtual image $O|R|^{2}$. Therefore, the hologram is filtered in a way to suppress the unwanted terms during reconstruction while maintaining the quality of the virtual image. To suppress unwanted terms we have to rewrite Eq.(16) as

$$
H=|O|^{2}+|R|^{2}+O \bar{R}+\bar{O} R .
$$

Considering Eq.(2), $O$ and $R$ can be substituted such that $O=$ $A_{O} e^{\mathbf{i} \varphi_{O}}$ and $R=A_{R} e^{\mathbf{i} \varphi_{R}}$, where $A_{O}$ is the amplitude of $O, \varphi_{O}$ is the phase of $O, A_{R}$ is the amplitude of $R$ and $\varphi_{R}$ is the phase of $R$ leading to

$$
\begin{aligned}
& H=A_{O}^{2}+A_{R}^{2}+\underbrace{A_{O} A_{R} e^{\mathbf{i} \varphi_{O}} e^{-\mathbf{i} \varphi_{R}}+A_{O} A_{R} e^{\mathbf{i} \varphi_{O}} e^{-\mathbf{i} \varphi_{R}}} \\
& H=A_{O}^{2}+A_{R}^{2}+\quad 2 A_{O} A_{R} \cos \left(\varphi_{O}-\varphi_{R}\right) .
\end{aligned}
$$

For better readability the $x$ and $y$ dependency of $H, A_{O}, A_{R}, \varphi_{O}$ and $\varphi_{R}$ have been omitted. The first two terms of Eq.(21) are close to be constant over the whole hologram, while the third term is statistically varying from $2 A_{O} A_{R}$ to $-2 A_{O} A_{R}$. Thus, the average intensity $\hat{H}$ over the whole hologram $H$ can be approximated by $\hat{H} \approx A_{O}^{2}+A_{R}^{2}$. The first two terms can therefore be suppressed by subtracting the average intensity $\hat{H}$ from the hologram $H$ leading to $H^{\prime}=H-\hat{H}$. If $H^{\prime}$ is used for reconstruction the reference term will be suppressed. This method is known as DC-term suppression in [31], since the almost constant term $A_{O}^{2}+A_{R}^{2}$ leads to the DC-term $\mathscr{H}(0,0)$, where $\mathscr{H}$ is the Fourier transform of the hologram $H$. Setting the reference wave sufficiently off-axis will deviate $\bar{O}$ such that it cannot be seen in front of the hologram.

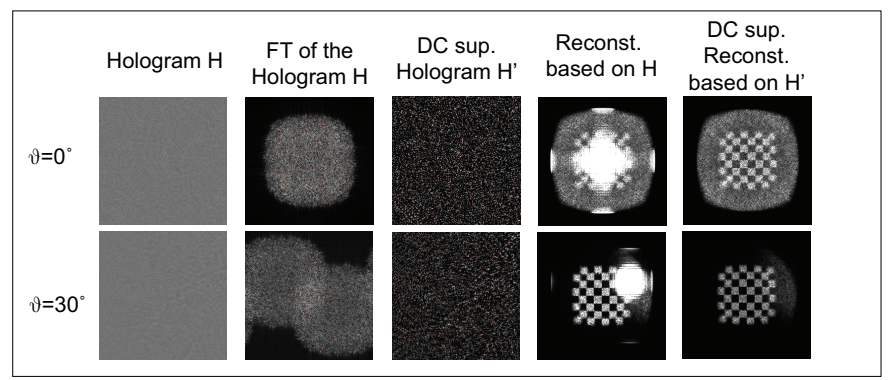

Fig. 7. Each row of pictures corresponds to a certain angle $\vartheta$. The DCTerm of the hologram $H$ gets suppressed leading to hologram $H^{\prime}$ depicted in the third column. In the reconstruction based on $H$ we can clearly see the reference term, while a reconstruction based on $H^{\prime}$ leads to much better results. Best results are obtained with a big angle $\vartheta$ leading however to a high sampling rate of the hologram.

A different method consisting of a high-pass filtering or asymmetrical masking of the hologram as presented in [30] would only improve our results if the spectra of $R, O$ and $\bar{O}$ would not overlap.

\section{IMAGE GENERATION}

Based on the propagation described in Sect. IV any wave field given on a synthetic object or on a reconstructed hologram can be evaluated on an aperture of an arbitrarily placed camera in space. The image generation consists of four steps, namely the simulation of an optical camera model Sect. VII-A, a multi-wavelength rendering for colored holograms Sect. VII$\mathrm{B}$, a depth evaluation for compositing of multiple objects Sect. VII-C and a speckle noise reduction Sect. VII-E. 


\section{A. Camera Model}

In a first step the simplest architecture of a camera, the pinhole camera, is studied for image generation. Artifacts occurring because of limited aperture size are described in Sect. VII-A while a more complex camera model including a lens is introduced in Sect. VII-A.

Pinhole Camera: As shown in Fig. 8a) a wave is propagating through a pinhole and producing an image on a parallel plane at distance $z$. Modeling the slit by an aperture $S_{A}$ and

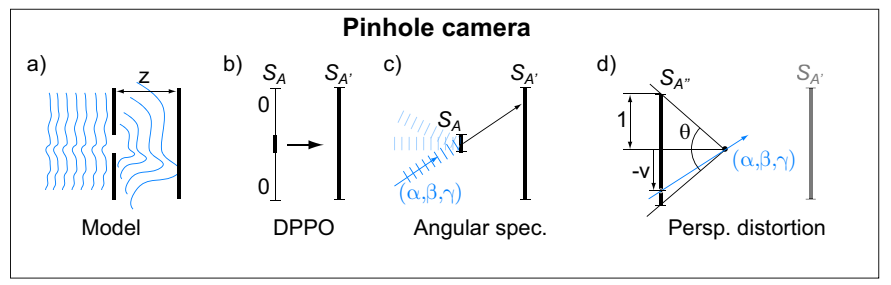

Fig. 8. Image generation simulating a pinhole camera as in a) can be achieved by DPPO b) or by making use of the directional cosines c). A perspective distortion leads to the final image d).

applying DPPO to propagate the wave to the image plane $S_{A^{\prime}}$ would be possible, but would require zero padding of $S_{A}$ (cf. Fig. 8b). Actually, assuming an indefinitely small slit and knowing the directional components $(\alpha, \beta, \gamma)$ of the wave front (cf.Sect. IV-C) is sufficient to generate the image at a distance $z$ assuming geometrical propagation from the slit (cf. Fig. 8c). The directional components can be calculated from the frequency components $v_{x}$ and $v_{y}$ of the angular spectrum as $(\alpha, \beta, \gamma)=\left(\lambda v_{x}, \lambda v_{y}, \sqrt{1-\left(\lambda v_{x}\right)^{2}-\left(\lambda v_{y}\right)^{2}}\right)$. Finally a mapping between the directional cosines and the image pixels given by coordinates $u$ and $v$ as in Fig. 8d) can be found by a projection matrix $P$ as $(\alpha, \beta, \gamma, 1) P \Rightarrow(u, v)$ leading to

$$
\left.\begin{array}{l}
\alpha=-\frac{u}{s_{x}}{\sqrt{\left(\frac{u}{s_{x}}\right)^{2}+\left(\frac{v}{s_{y}}\right)^{2}+1}}^{-1} \\
\beta=-\frac{v}{s_{y}} \sqrt{\left(\frac{u}{s_{x}}\right)^{2}+\left(\frac{v}{s_{y}}\right)^{2}+1}-1
\end{array}\right\} s_{y}=\frac{1}{\tan \left(\frac{\theta}{2}\right)}, s_{x}=r_{a} s_{y}
$$

$\theta$ is the field of view in $y$ direction with the aspect ratio $r_{a}=\frac{w}{h}$ where $w$ is the image width and $h$ the image height. The pixels of the image are computed by a weighted sum (e.g. bilinear interpolation) of the directional cosines.

Resolution The maximal resolution of the image is determined by the size of the pinhole $W$. This can be shown by the relation of the spacing $\delta_{f}$ in the frequency and the spacing $\delta$ in the spacial domain given as $\delta \delta_{f}=\frac{1}{N}$ where $N$ is the number of samples. The smallest non-zero frequency is given as $\delta_{f}=\frac{1}{W}$, since $W=N \delta$. This corresponds to the direction cosine $\alpha_{\text {min }}=\frac{\lambda}{W}$ and gets close to the Rayleigh resolution criterion $\alpha=1.22 \frac{\lambda}{D}$ describing the critical angle at which two point sources can still be distinguished when diffracting at a circular aperture of diameter $D$ (see [37]).

Apodization: The effect of a rectangular aperture as used in our pipeline can be simulated as a multiplication by a rect-function in the spatial domain and as a convolution with a sinc-function in the frequency domain (cf. Fig. 9a). The convolution with the sinc-function can lead to visible artifacts called ringing appearing as blur in the axial directions. This blur can be minimized by applying a tapering function as proposed in [38] and [39]. In our implementation we use a cosine window function being 1 in the middle of the aperture and decreasing to 0 at the border of the aperture resulting in an apodization as shown in Fig. 9b).
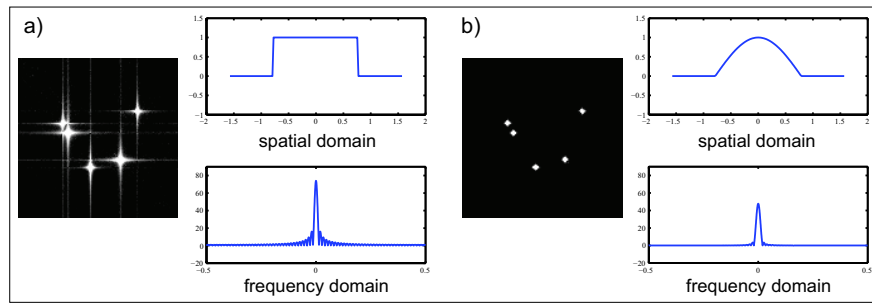

Fig. 9. Applying a rect-function for the aperture leads to ringing artifacts a). The Fourier transform of the aperture is shown below. Multiplying an apodization function to the aperture in the spatial domain results in an image free of ringing $b$ ).

Thin Lens: Placing the camera at arbitrary positions while choosing the object appearing in focus requires a lens. We use a thin lens model:

$$
\frac{1}{d_{O}}+\frac{1}{d_{i}}=\frac{1}{f}
$$

with $d_{O}$ being the distance from the lens to the object, $d_{i}$ being the distance from the lens to the image and $f$ being the focal length.

The effect of a thin lens can be simulated by multiplying a complex-valued function $L(x, y)$

$$
L(x, y)=e^{-\mathbf{i} k r}, \text { with } r=\sqrt{x^{2}+y^{2}+f^{2}}
$$

inducing a phase shift to the wavefront [17]. The phase shift transforms the spherical wave with origin at focal distance $f$ into an almost planar wave:

$$
\left(A_{0} \frac{e^{\mathbf{i} k r}}{r}\right) L(x, y)=\frac{A_{0}}{r} .
$$

Even though it does not lead to a perfect planar wave it results in a good approximation for a point source being far away compared to the size of the lens. Figure 10 shows a scene consisting of five points being placed at different distances from the lens taken with three different focal lengths. Off-

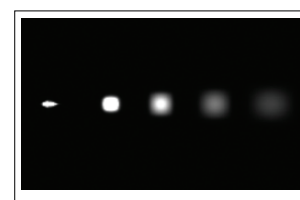

$f=60$

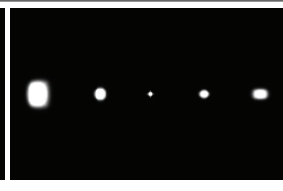

$f=100$

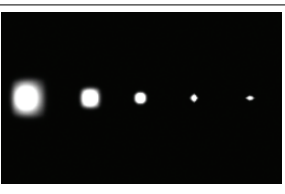

$f=140$
Fig. 10. Scene with five points placed at different depth and rendered with three different focal lengths $f$.

axis points at the edge of the image can produce a cometshaped image, an effect known as coma aberration. For more general applications one would need to implement a lens simulation, which distributes aberration and artifacts over the whole image.

Due to a physically based model of the holography pipeline effects like depth of field or refocusing are automatically generated (cf.Sect. VIII). 


\section{B. Color holograms}

A non-monochromatic wave could be split into all spectral frequencies, which could be propagated separately in the wave framework. However, instead of simulating all frequencies separately we chose three primary colors $\left(\lambda_{r}, \lambda_{g}, \lambda_{b}\right.$, having approximately the wavelength of red, green and blue) from which other visible colors can be combined linearly. The amplitudes $A_{0}$ at the origin of each wave are set as $A_{0}=\sqrt{I}$, where $I$ is the intensity of one of the primary colors. The final image can thus be rendered by calculating the scene for each monochromatic primary color once.

Simulating non-monochromatic colors introduces another artifact called chromatic aberration when using lenses in the optical path. This means that when choosing a focal length $f_{r}$ for a lens, the effective focal length for the image generated with wavelength $\lambda_{g}$ is $f_{r}\left(\frac{\lambda_{g}}{\lambda_{r}}\right)$. Applying a Fourier based image generation and perspective distortion does not introduce further chromatic artifacts.

When choosing the wavelength for the primary colors we are not restricted to take realistic values since the intensity $I$ of the color channel only influences the amplitude. The wavelength $\lambda_{r}, \lambda_{g}$ and $\lambda_{b}$ could be exactly the same avoiding all aberrations and artifacts related to wavelength differences. In physical lens systems, multiple lenses and lens materials are minimizing the effect of chromatic aberration.

\section{Evaluate Depth}

So far we have shown the generation of an image of a single object. However, if we have scenes composed of several objects occlusions have to be taken care of. We present a depth reconstruction from a hologram in order to compose objects using the depth buffer.

Depth From Phase: Calculating depth from phase is a straightforward approach also used in digital holographic interferometry as shown in [15]. Knowing the phase of the source and the image, the relative depth can be calculated out of this phase difference modulo $\lambda$. In order to unambiguously reconstruct the surface the depth difference of two neighboring pixels has to be smaller than $\frac{\lambda}{2}$. However, a relative surface is not sufficient for depth composition.

Depth From Phase Difference: A more advanced approach consists in generating several images with slightly different wavelengths $\lambda_{i}$. In our case we generate two images with two wavelengths $\lambda_{1}$ and $\lambda_{2}$ being related by $\lambda_{1}=\varepsilon \lambda_{2}$ with $\varepsilon<1$. The phase $\varphi_{i}$ can be calculated as $\varphi_{i}=\left(\frac{2 \pi}{\lambda_{i}} * r+\varphi_{0}\right) \bmod \pm \pi$ where $\varphi_{i} \bmod \pm \pi=\left(\left(\varphi_{i}+\right.\right.$ $\pi) \bmod 2 \pi)-\pi$. This leads to a phase difference $\Delta \varphi$ in $[0,2 \pi)$ as $\Delta \varphi=2 \pi r\left(\frac{1}{\lambda_{1}}-\frac{1}{\lambda_{2}}\right)$. Considering the maximal phase difference $2 \pi$ we get the maximal radius $r_{\max }$ as

$$
\begin{aligned}
r_{\text {max }} & =\frac{2 \pi}{2 \pi\left(\frac{1}{\varepsilon \lambda_{1}}-\frac{1}{\lambda_{1}}\right)}, \text { with } \\
\varepsilon & =\frac{1}{1+\frac{\lambda_{1}}{r_{\max }}} .
\end{aligned}
$$

Therefore, the bigger $r_{\max }$ is, the closer $\varepsilon$ has to be to 1 leading to a small difference between $\lambda_{1}$ and $\lambda_{2}$. Applying this depth reconstruction results in an absolute depth needed for depth composition. Before having a closer look at the influence of interference problems in Sect. VII-C, we will analyze the impact of a lens on depth reconstruction.

Depth Reconstruction Using A Lens: Applying a lens for image generation imposes a consideration of influence on depth reconstruction as well. Considering the mathematical representation of a lens with focal length $f$ as described in Eq.(24) we can find images $U_{\lambda_{1}}(P), U_{\lambda_{2}}(P)$ at distance $R$ from the origin as follows:

$$
U_{\lambda_{j}}=A_{0} \frac{e^{\mathbf{i} k_{j} R}}{R} e^{-\mathbf{i} k_{j} f} \quad, \text { where } k_{j}=\frac{2 \pi}{\lambda_{j}}, j=1,2 .
$$

The phase difference $\Delta \varphi$ evaluates to

$$
\Delta \varphi=2 \pi\left(\frac{1}{\lambda_{2}}-\frac{1}{\lambda_{1}}\right)\left(r_{f}-f\right)
$$

for the phases given as $\varphi_{1}=k_{1}\left(r_{f}-f\right)$ and $\varphi_{2}=k_{2}\left(r_{f}-f\right)$. The distance of the source can thus be found by solving Eq.(29) for $r_{f}$. Comparing the reconstruction based on a camera with a lens leading to depth $r_{f}$ with the reconstruction without a lens leading to depth $r$ we find the simple relation to be $r_{f}=r+f$. In order to find the desired distance values $d_{\max }$ around the focal point $\left[f-d_{\max }, f+d_{\max }\right]$ we have to evaluate a new factor $\varepsilon_{l}$ such that $\lambda_{2}=\varepsilon_{l} \lambda_{1}$ to:

$$
\varepsilon_{l}=\frac{1}{1+\frac{\lambda_{1}}{2 d_{\max }}} \text {. }
$$

Influence Of Interference: Despite of having better results using a depth reconstruction approach based on phase difference, we still have the problem of interference. We explain the case of two interfering point sources $P S_{1}$ and $P S_{2}$ with origins $P_{1}$ and $P_{2}$ at distance $r_{1}$ and $r_{2}$ from the point of evaluation $P^{\prime}$ (cf. Fig. 11a) and finally infer the general case of possible multiple point sources in the end. Since $r_{1}$ and $r_{2}$ cannot be reconstructed separately we would like to find a depth $r^{\prime}$ which lies in between the two distance values such that $r_{1} \leq r^{\prime} \leq r_{2}$. This is equal to guaranteeing $\Delta \varphi_{1} \leq \Delta \varphi^{\prime} \leq \Delta \varphi_{2}$ for $\Delta \varphi_{1}$ and $\Delta \varphi_{2}$ being the individual phase differences of $P S_{1}$ and $P S_{2}$ respectively and $\Delta \varphi^{\prime}$ being the phase difference of the superposition of $P S_{1}$ and $P S_{2}$ at $P^{\prime}$.

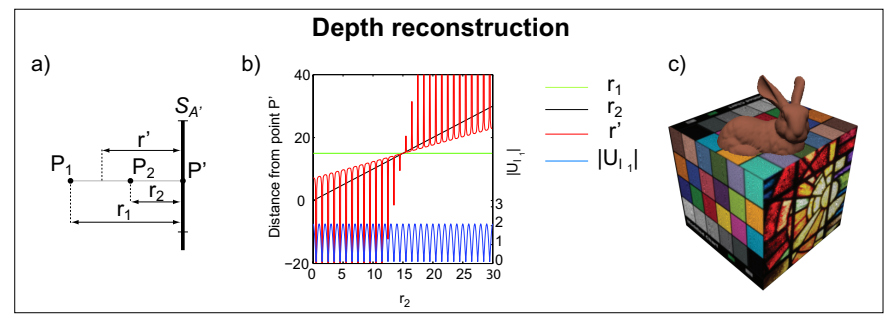

Fig. 11. Moving $P_{2}$ in a) from $P^{\prime}$ to $r_{2}=30$ is leading to a reconstruction of $r^{\prime}$ as in b). Improving results in b) by multiple iterations for depth refinement leads to results as in $\mathrm{c}$ ).

The complex valued image $U_{\lambda_{j}}$ describes the interference pattern of $P S_{1}$ with amplitude $A_{1_{0}}$ and $P S_{2}$ with amplitude $A_{2_{0}}$ with wavelength $\lambda_{j}$ as follows:

$$
U_{\lambda_{j}}=A_{1_{0}} \frac{e^{\mathbf{i} k_{j} r_{1}}}{r_{1}}+A_{2_{0}} \frac{e^{\mathbf{i} k_{j} r_{2}}}{r_{2}} \quad \text {, with } k_{j}=\frac{2 \pi}{\lambda_{j}} \text {. }
$$




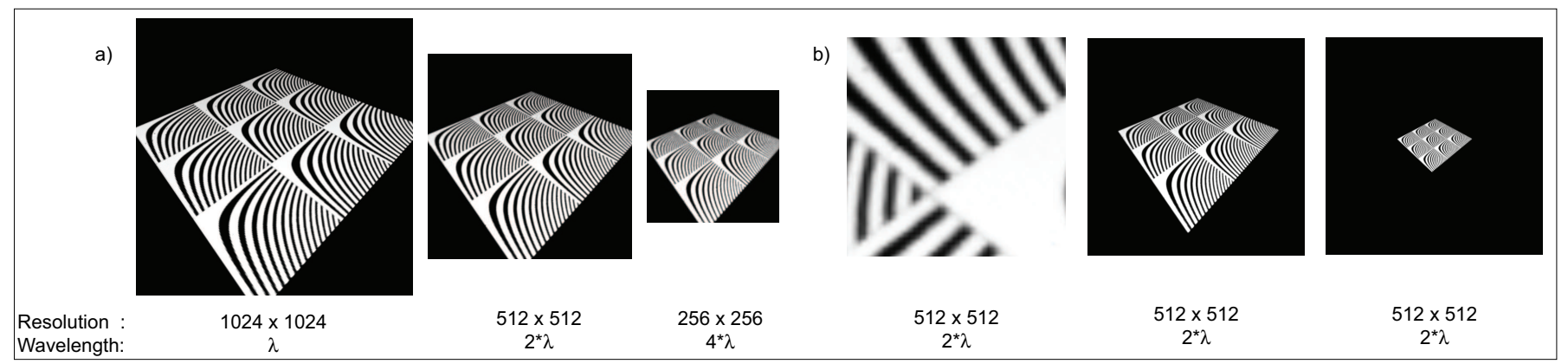

Fig. 12. a) The three images show the same scene evaluated at different image resolutions and therefore different wavelengths. b) shows different magnifications of the same textured plane without having to apply any filtering. (Please look at the pdf for correct anti aliasing appearance.)

Ignoring interference would lead to a depth reconstruction as seen in Fig. 11b). The desirable values of $r^{\prime}$ lying in between $r_{1}$ and $r_{2}$ always coincide with the maxima of $\left|U_{\lambda_{1}}\right|$. This can be shown since $\left|U_{\lambda_{1}}\right|=\max \left(\left|U_{\lambda_{1}}\right|\right)$ implies $\min \left(\Delta \varphi_{1}, \Delta \varphi_{2}\right) \leq$ $\Delta \hat{\varphi} \leq \max \left(\Delta \varphi_{1}, \Delta \varphi_{2}\right)$. The associative property of vector addition allows the extension of two point sources to multiple point sources, so the proof holds for the addition of a new point source with the sum of the remaining point sources as well. In order to improve depth quality in case of $\left|U_{\lambda_{1}}\right| \neq \max \left(\left|U_{\lambda_{1}}\right|\right)$ the image is rendered several times while randomizing the phase of the point sources for every iteration. Depending on the number of iterations the depth reconstruction can be arbitrarily close to an optimal $r^{\prime}$.

Finally, the depth buffer is multiplied by a mask created by a thresholding of an introduced opacity channel, verifying that a pixel belongs to a certain object. Note, that once the wave based object has been evaluated the compositing with rasterized objects as depicted in Fig. 11c) can be performed in real time.

\section{Aliasing free images}

A rasterized image is essentially a discrete function defined on a 2D regular grid. To generate an aliasing free image the sampling process has to satisfy the Nyquist criterion. Since the Nyquist criterion in traditional computer graphics depends on the frequency of the projected textures, appropriate filtering has to be applied to obtain anti aliased images as has been shown by Heckbert [40][41]. However, in our pipeline generated images are inherently free of aliasing.

The scenes used in our pipeline can consist of point primitives or textured planar primitives emitting waves of equal wavelength $\lambda$. The color of the point sources as well as the planar primitives is encoded in the amplitude of the propagating waves. The interference generated by the superposition of all these primary waves create a wavefront containing the complete information of the whole scene. Since the wavefront does not have a higher frequency than the interference pattern, the sampling rate will not have to be higher either. In order to avoid any aliasing the sampling rate of the interference pattern has to guarantee the Nyquist frequency and therefore a sampling distance of $\frac{\lambda}{2}$, which has been shown to be the case in Sect. VI-A. By simulating monochromatic waves we implicitly guarantee a distinct lower bound for the wavelength $\lambda$ and therefore a distinct upper bound for the required sampling rate $\frac{2}{\lambda}$.

Finally, by Fourier transforming the sampled interference pattern we get the aliasing free directional components of the wavefront. After an according mapping described in Sect. VIIA we get the aliasing free image.

Changing image resolution implies a modified frequency in the sampling process of the image function. In traditional computer graphics this requires a pre- or post-filtering of the texture in order to comply with the Nyquist frequency. In our pipeline, however, a lower image resolution does not require any filtering, but simply an increased wavelength of the monochromatic source. In order to avoid a scaling of the object wave the hologram is regenerated using the new wavelength. Fig. 12a shows three renderings with different resolutions and therefore three different wavelengths. We did not apply any filtering on the original texture and still obtain aliasing free images. Furthermore, magnification or minification does not require any filtering or wavelength adjustments as can be seen in Fig. 12b.

\section{E. Image improvement}

Throughout our pipeline, coherence is a fundamental property to generate a standing wave which again is necessary to record and reconstruct a hologram. However, generating images based on coherent light can cause visible artifacts due to the static interference pattern called speckle noise, which can even be visible by the naked eye in physical setups as shown in [42].

Speckle Noise Reduction Through Higher Resolution: The speckle size $\delta_{S}$ depends on the distance $x$ of two interfering light waves with wavelength $\lambda$ being at distance $z$ of the interference measurement as follows (see [15]):

$$
\delta_{S}=\frac{\lambda z}{x}
$$

Therefore the minimal speckle size $\delta_{S_{\min }}$ can be calculated dependent on the aperture size $W$ as $\delta_{S_{\min }}=\frac{\lambda z}{W}$.

These properties imply a higher frequency of speckle noise with an increasing image resolution meaning a bigger $W$ (cf. Sect. VII-A), whereas the frequency of the actual image content stays the same. Hence, filtering out high frequency speckle noise does not affect the actual image content of the 
rendered image. Additional reduction can be achieved by down sampling the image using a low pass filter.

Speckle Noise Reduction By Temporal Averaging: Instead of filtering in space as in Sect. VII-E it might be faster to filter in time by rendering multiple images from a diffuse object with randomized phases. Computing the final image is achieved by either blending all the images or choosing the maximal value per pixel. The SNR is better for the maximal composition of $N$ images if only two sources with amplitude $A_{1}$ and $A_{2}$ are contributing to an image point $P$ of image $k$ with intensity $I_{k}(P)$, since the expectation $E\left[\lim _{N \rightarrow \infty} \max _{k}\left(I_{k}(P)\right)\right]=\left(A_{1}+A_{2}\right)^{2}$ is bigger than the expectation for averaging $E\left[\frac{1}{N} \sum_{k=1}^{N} I_{k}(P)\right]=A_{1}^{2}+A_{2}^{2}$. However, the more interfering sources are involved the smaller the probability that the maximal value is found after $N$ iterations. The necessary and sufficient condition for a maximal composition of interfering sources consists in all sources having equal phases as shown in Sect. VII-C.

\section{RESULTS}

Using our framework we are capable of rendering high quality images, while taking into account depth of field and refocusing of the scene by simply adjusting the aperture size or focal length as if a real camera would have been used (cf. Fig. 13). Even big objects with primitives count up to $300 \mathrm{~K}$ (cf. Fig. 14f) can be evaluated in a reasonable amount of time (cf. Tab. I), due to usage of fragment shaders and angular spectrum propagation. By using propagation in frequency space, time can be reduced considerably. A $1024^{2}$ hologram as used in Fig. 14e) can be propagated in 50s instead of $5 \mathrm{~min} 30 \mathrm{~s}$. Creating a hologram of $1024^{2}$ with double precision per primary color channel leads to a total size of $24 \mathrm{MB}$. Undersampling of a high-frequency texture during the rasterization step in ray-based image generation can lead to severe aliasing artifacts whereas in a wave-based framework anti-alising comes for free Fig. 15c). All the images were generated on a Pentium $43.2 \mathrm{GHz}$ containing a NVidia GeForce 7800GT. Currently we make use of fragment shaders for point evaluations.

TABLE I

STATISTICS

\begin{tabular}{|c|c|c|c|c|c|}
\hline Figure & $\begin{array}{c}\text { \# Of } \\
\text { Prim. }\end{array}$ & $\begin{array}{c}\text { Holo. } \\
\text { Size }\end{array}$ & $\begin{array}{c}\text { Pict. } \\
\text { Size }\end{array}$ & $\begin{array}{c}\text { Time } \\
\text { per It. }\end{array}$ & $\begin{array}{c}\text { \# Of } \\
\text { It. }\end{array}$ \\
\hline Fig. 13 & $100 \mathrm{~K}$ & $1024^{2}$ & $1024^{2}$ & $185 \mathrm{~s}$ & 70 \\
\hline Fig. 14a)-d) & $50 \mathrm{~K}$ & $512^{2}$ & $512^{2}$ & $43 \mathrm{~s}$ & 24 \\
\hline Fig. 14e) & $50 \mathrm{~K}$ & $1024^{2}$ & $1024^{2}$ & $155 \mathrm{~s}$ & 74 \\
\hline Fig. 14f) & $300 \mathrm{~K}$ & $1024^{2}$ & $1024^{2}$ & $325 \mathrm{~s}$ & 70 \\
\hline Fig. 15a)/d) & $137 \mathrm{~K}$ & $1024^{2}$ & $1024^{2}$ & $197 \mathrm{~s}$ & 90 \\
\hline Fig. 15b) & $173 \mathrm{~K}$ & $1024^{2}$ & $1024^{2}$ & $241 \mathrm{~s}$ & 90 \\
\hline
\end{tabular}

\section{DisCUSSION AND FUture WORK}

We presented a framework for holographic scene representation and rendering that integrates smoothly into conventional graphics architectures. The wave nature of holographic image generation provides a variety of interesting features including depth of field and high quality antialiasing. The

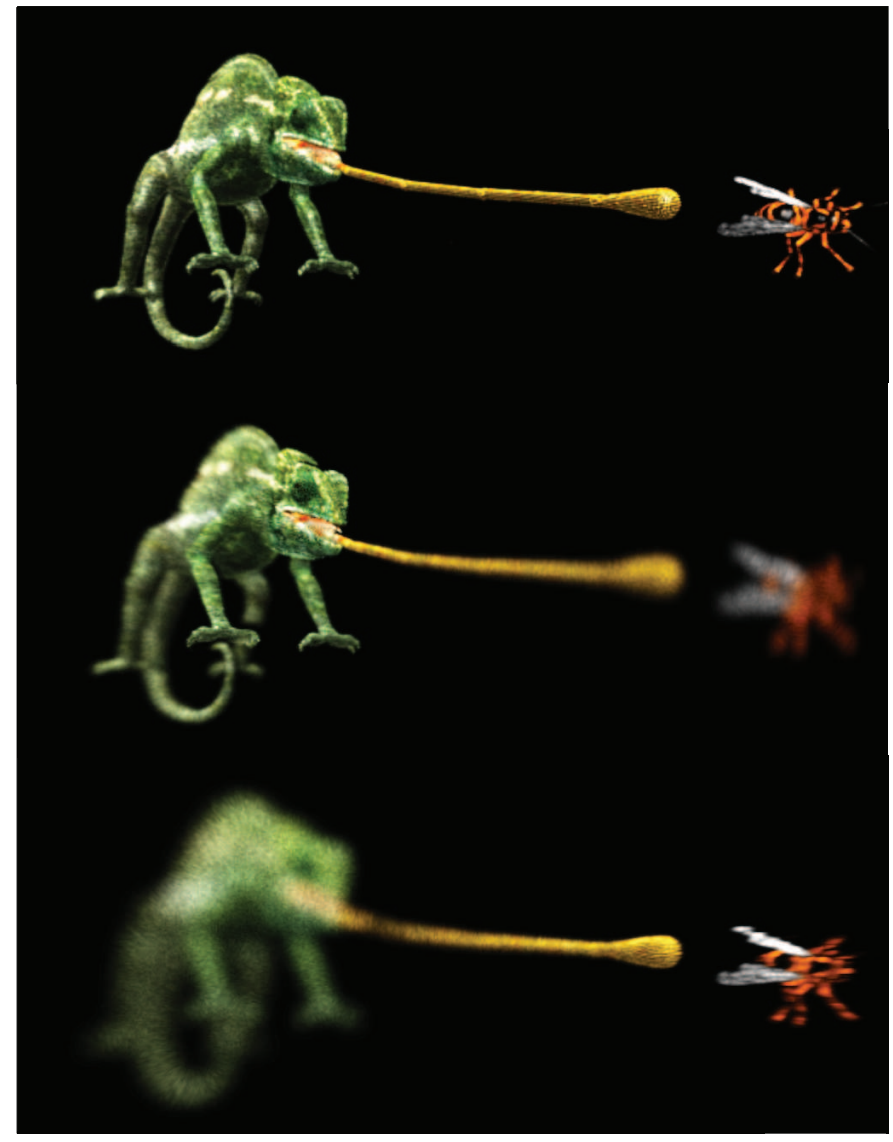

Fig. 13. The top scene has been calculated with a small aperture leading to a large depth of field. The two images below were computed using a bigger aperture and two different focal lengths.

phase information inherently embedded into the representation allows us to reliably reconstruct view-dependent depth and to compose digital holograms with standard 2D framebuffer graphics. While holograms require a very high sampling rate in theory, our practical implementation computes high quality renderings from reasonably sized digital holograms.

In spite of hardware acceleration, holographic image generation is still far from real-time, which sets one of the limitations of the presented method. In future work, we plan to explore the parallel nature of wave evaluation to further accelerate the rendering. In addition, relighting, reshading, and deformation operators in wave domain are of highest interest to us. Finally, efficient compression of digital holograms could be achieved by exploiting their spectral composition. 


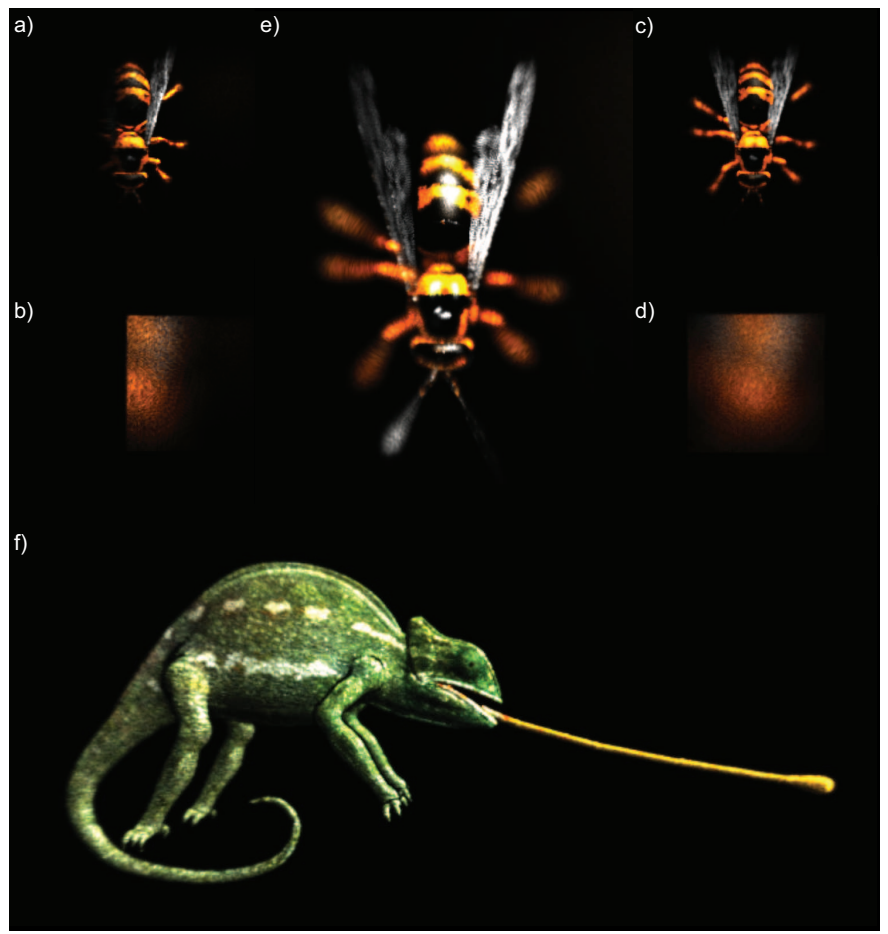

Fig. 14. a)b) and c)d) are pairs of images of the same scene rendered with different foci. b) and d) are images focused on the holographic plate revealing its rectangular shape. Using a bigger hologram in e) avoids evanescence of the object at the border of the hologram. The chameleon in $f$ ) is rendered with a bigger depth of field than the wasp in e).

\section{REFERENCES}

[1] M. Levoy and P. Hanrahan, "Light field rendering," Computer Graphics, vol. 30, no. Annual Conference Series, pp. 31-42, 1996. [Online]. Available: citeseer.ist.psu.edu/levoy96light.html

[2] D. N. Wood, D. I. Azuma, K. Aldinger, B. Curless, T. Duchamp, D. H. Salesin, and W. Stuetzle, "Surface light fields for 3d photography," in SIGGRAPH 'O0: Proceedings of the 27th annual conference on Computer graphics and interactive techniques. New York, NY, USA: ACM Press/Addison-Wesley Publishing Co., 2000, pp. 287-296.

[3] A. Isaksen, L. McMillan, and S. J. Gortler, "Dynamically reparameterized light fields," in SIGGRAPH '00: Proceedings of the 27th annual conference on Computer graphics and interactive techniques. New York, NY, USA: ACM Press/Addison-Wesley Publishing Co., 2000, pp. 297-306.

[4] S. J. Gortler, R. Grzeszczuk, R. Szeliski, and M. F. Cohen, "The lumigraph," Computer Graphics, vol. 30, no. Annual Conference Series, pp. 43-54, 1996. [Online]. Available: citeseer.ist.psu.edu/gortler96lumigraph.html

[5] C. Buehler, M. Bosse, L. McMillan, S. Gortler, and M. Cohen, "Unstructured lumigraph rendering," in SIGGRAPH '01: Proceedings of the 28th annual conference on Computer graphics and interactive techniques. New York, NY, USA: ACM Press, 2001, pp. 425-432.

[6] P. Debevec, T. Hawkins, C. Tchou, H.-P. Duiker, W. Sarokin, and M. Sagar, "Acquiring the reflectance field of a human face," in SIGGRAPH '00: Proceedings of the 27th annual conference on Computer graphics and interactive techniques. New York, NY, USA: ACM Press/Addison-Wesley Publishing Co., 2000, pp. 145-156.

[7] W. Matusik, H. Pfister, A. Ngan, P. A. Beardsley, R. Ziegler, and L. McMillan, "Image-based 3d photography using opacity hulls," ACM Trans. Graph., vol. 21, no. 3, pp. 427-437, 2002.

[8] T. Weyrich, "Rendering deformable surface reflectance fields," IEEE Transactions on Visualization and Computer Graphics, vol. 11, no. 1, pp. 48-58, 2005, senior Member-Hanspeter Pfister and Senior MemberMarkus Gross.

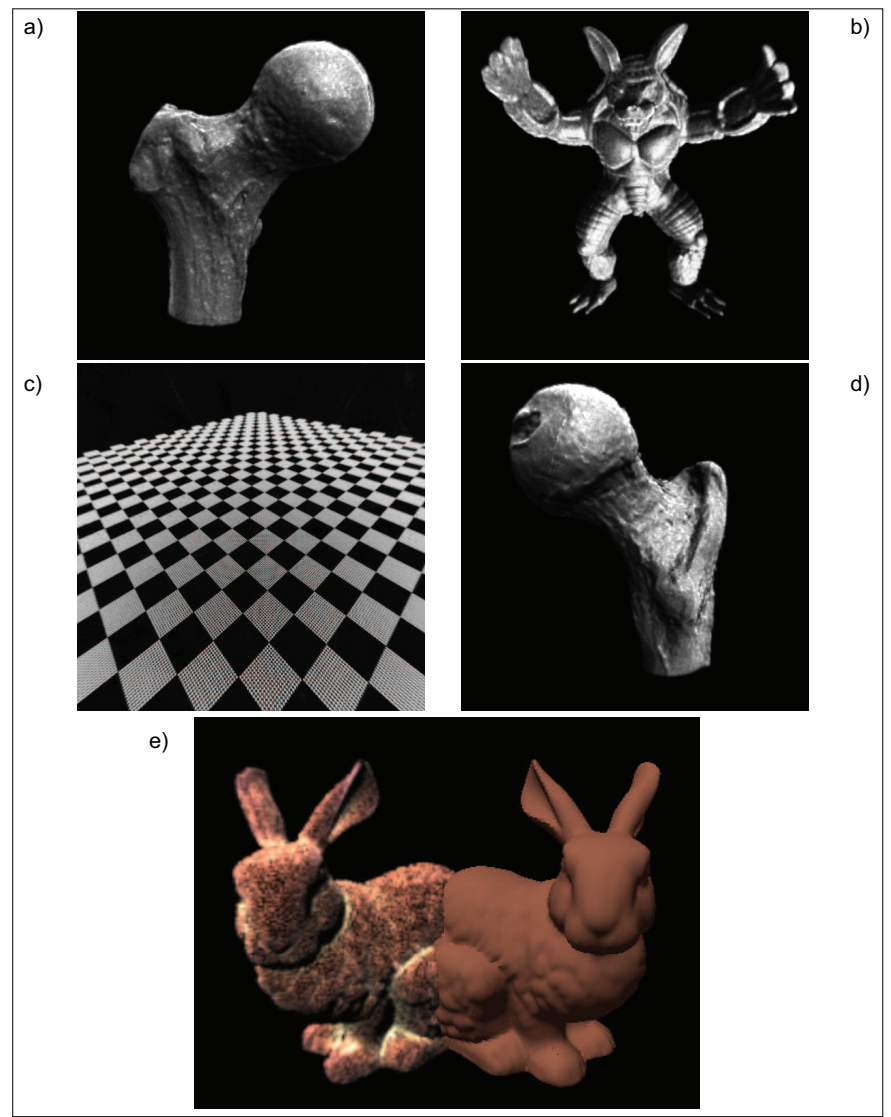

Fig. 15. Wave-based evaluation of point-based objects can represent surfaces of high geometric detail, as long as the sampling of the point-based object is dense enough in the first place. On the lower left c) an aliasing-free checkerboard generated using our pipeline is shown. e) is showing a scene composed of a holographic and a mesh based representation of the same object.

[9] T. Okoshi, Three-Dimensional Imaging Techniques. New York: Academic Press, 1976.

[10] S. A.Benton, Selected Papers on Three-Dimensional Displays. SPIE Int'l Soc. for Optical Eng., 2001.

[11] C. Slinger, C. Cameron, and M. Stanley, "Computer-generated holography as a generic display technology," Computer, vol. 38, no. 8, pp. 46-53, 2005.

[12] M. Lucente and T. A. Galyean, "Rendering interactive holographic images," in SIGGRAPH '95: Proceedings of the 22nd annual conference on Computer graphics and interactive techniques. New York, NY, USA: ACM Press, 1995, pp. 387-394.

[13] M. Lucente, "Interactive three-dimensional holographic displays: seeing the future in depth," SIGGRAPH Comput. Graph., vol. 31, no. 2, pp. 63-67, 1997.

[14] M. Halle and A. Kropp, "Fast computer graphics rendering for full parallax spatial displays," 1997. [Online]. Available: citeseer.csail.mit.edu/halle97fast.html

[15] U. Schnars and W. Jüptner, Digital Holography : digital hologram recording, numerical reconstruction, and related techniques. Springer, 2005.

[16] D. Gabor, “A new microscope principle," Nature, vol. 161, pp. 777-779, 1948.

[17] J. W. Goodman, Introduction to Fourier Optics. San Francisco: McGraw-Hill Book Company, 1968

[18] T. Tommasi and B. Bianco, "Frequency analysis of light diffraction between rotated planes," Optics Letters, vol. 17, pp. 556-558, Apr. 1992.

[19] — , "Computer-generated holograms of tilted planes by a spatial frequency approach,” J. Opt. Soc. Am. A, vol. 10, pp. 299-305, Feb. 1993. 
[20] K. Matsushima, H. Schimmel, and F. Wyrowski, "Fast calculation method for optical diffraction on tilted planes by use of the angular spectrum of plane waves," Optical Society of America Journal A, vol. 20, pp. 1755-1762, Sept. 2003.

[21] N. Delen and B. Hooker, "Free-space beam propagation between arbitrarily oriented planes based on full diffraction theory: a fast Fourier transform approach," J. Opt. Soc. Am. A, vol. 15, pp. 857-867, April 1998.

[22] F. Durand, N. Holzschuch, C. Soler, E. Chan, and F. Sillion, "A frequency analysis of light transport," ACM Transactions on Graphics (Proceedings of the SIGGRAPH conference), vol. 24, no. 3, aug 2005, proceeding. [Online]. Available: http://artis.imag.fr/Publications/2005/DHSCS05

[23] K. Matsushima, "Computer-generated holograms for three-dimensional surface objects with shade and texture," Applied Optics, vol. 44, pp. 4607-4614, August 2005.

[24] K. Matsushima, "Exact hidden-surface removal in digitally synthetic full-parallax holograms," in Proc. SPIE Vol. 5742, p. 53-60, Practical Holography XIX: Materials and Applications, June 2005, pp. 25-32.

[25] T. Totsuka and M. Levoy, "Frequency domain volume rendering," Computer Graphics, vol. 27, no. Annual Conference Series, pp. 271-278, 1993. [Online]. Available: citeseer.ist.psu.edu/totsuka93frequency.html

[26] S. Yusuke, I. Masahide, and Y. Toyohiko, "Color computer-generated holograms from projection images," OPTICS EXPRESS, vol. 12, pp. 2487-2493, May 2004.

[27] K. Matsushima and M. Takai, "Recurrence Formulas for Fast Creation of Synthetic Three-Dimensional Holograms," Applied Optics, vol. 39, pp. 6587-6594, Dec. 2000.

[28] M. Koenig, O. Deussen, and T. Strothotte, "Texture-based hologram generation using triangles," in Proc. SPIE Vol. 4296, p. 1-8, Practical Holography XV and Holographic Materials VII, Stephen A. Benton; Sylvia H. Stevenson; T. John Trout; Eds., June 2001, pp. 1-8.

[29] A. Ritter, J. Böttger, O. Deussen, M. König, and T. Strothotte, "Hardware-based Rendering of Full-parallax Synthetic Holograms," Applied Optics, vol. 38, pp. 1364-1369, February 1999.

[30] E. Cuche, P. Marquet, and C. Depeursinge, "Spatial filtering for zeroorder and twin-image elimination in digital off-axis holography," Applied Optics, vol. 39, pp. 4070-4075, August 2000.

[31] T. Kreis and W. Jüptner, "Suppression of the dc term in digital holography," Opt. Eng., vol. 36, pp. 2357-2360, August 1997.

[32] U. Schnars and W. Jüptner, "Digital recording and numerical reconstruction of holograms," Meas. Sci. Technol., vol. 13, pp. 85-101, 2002.

[33] V. Vaish, B. Wilburn, N. Joshi, and M. Levoy, "Using plane + parallax for calibrating dense camera arrays." in CVPR (1), 2004, pp. 2-9.

[34] M. Levoy, B. Chen, V. Vaish, M. Horowitz, I. McDowall, and M. Bolas, "Synthetic aperture confocal imaging," ACM Trans. Graph., vol. 23, no. 3, pp. 825-834, 2004.

[35] R. Ng, "Fourier slice photography," ACM Trans. Graph., vol. 24, no. 3, pp. 735-744, 2005.

[36] J. A. Ratcliffe, "Some aspects of diffraction theory and their application to the ionosphere," Reports on Progress in Physics, vol. 19, no. 1, pp. 188-267, 1956. [Online]. Available: http://stacks.iop.org/00344885/19/188

[37] P. A. Tipler, Physics for Scientists and Engineers, Third Edition, Extended Version. Worth Publishers, Inc., 1991.

[38] G. Gbur, OPTI 6271/8271 Lecture Notes Spring 2005. UNCC, January 2005.

[39] G. Gbur and P. Carney, "Convergence criteria and optimization techniques for beam moments," Pure and Applied Optics, vol. 7, pp. 12211230, September 1998.

[40] P. S. Heckbert, "Fundamentals of texture mapping and image warping," Master's thesis, 1989. [Online]. Available: citeseer.ist.psu.edu/150876.html

[41] N. Greene and P. S. Heckbert, "Creating raster omnimax images from multiple perspective views using the elliptical weighted average filter," IEEE Comput. Graph. Appl., vol. 6, no. 6, pp. 21-27, 1986.

[42] J. Dainty, A. Ennos, M. Françon, J. Goodman, T. McKechnie, and G. Parry, Laser Speckle. New York: Springer-Verlag, 1975. 